\title{
Der Runenstein von Tanum \\ - ein religionsgeschichtliches Denkmal \\ aus urnordischer Zeit
}

\author{
Von OTtar GrøNVIK
}

\section{Der Fundort}

1.1. Der Runenstein von Tanum (KJ 61) ist der wissenschaftlichen Welt schon seit dem Anfang des vorigen Jahrhunderts bekannt; er wurde zum ersten Mal 1823 publiziert (vgl. Stephens 1866-84, 1, 196). ${ }^{1}$ Damals lag er mit anderen Steinen zusammen als Steg über einem kleinen Wasserlauf mit der Inschriftseite nach oben, an einem kleinen Weg zwischen den Gehöften Kalleby und Trättelanda im Kirchspiel Tanum im nördlichen Bohuslän (vgl. Boije 1886, 259; Friesen 1924, 130). Im Jahre 1866 wurde er etwa $6 \mathrm{~km}$ nordwärts auf den Platz vor der Kirche von Tanum überführt.

1.2. Die ursprüngliche Lage des Steines ist nicht sicher zu ermitteln. Anders Bæksted sprach die Vermutung aus, daß der Stein ursprünglich gerade dort errichtet worden sei, wo er später gefunden wurde, "am Schnittpunkt zwischen Weg und Wasserlauf" (Bæksted 1951, 83). ${ }^{2}$ Dabei dachte er an einen Gedenkstein ohne Verbindung mit einer Grabanlage (Bæksted 1951, 87). Andere glauben dagegen, daß der Stein an oder über einem Grab gestanden hat, wobei sie sich vor allem auf ihre sprachliche Deutung der Inschrift berufen (Friesen 1924, 134; Nordén 1934, 103; Marstrander 1952, 202). Archäologisch ist die Frage nicht zu entscheiden (Krause 1966, 139); die Theorie von einer Grabanlage hat aber eine gewisse Stütze darin, daß viele urnordische Runensteine gerade an, auf oder in Gräbern gefunden worden sind.

1 KJ 61 gibt die Nummer der betreffenden Inschrift in Krause 1966 an.

2 Dieses und alle übrigen in dieser Abhandlung angeführten Zitate von nordischen Verfassern sind von mir (O. G.) ins Deutsche übertragen. 


\section{Die Runen}

2.1. Der Stein ist ein mächtiger Block, fast $3 \mathrm{~m}$ hoch, ungefähr $1,5 \mathrm{~m}$ breit und 13-40 cm dick. Die Vorderseite hat die Form eines plumpen Vierecks, dessen Oberseite unregelmäßig nach links oben ansteigt (siehe Fotos bei Friesen 1924, 128 f.; Marstrander 1952, 197; Krause 1966, Tafel 27).

Die Runen gehen linksläufig von unten nach oben und bilden zusammen eine ungefähr $1,25 \mathrm{~m}$ lange Zeile, die sich längs der Mittelachse des Steins bis an die Steinkante oben hinzieht. Die Runen sind sämtlich vorzüglich erhalten und können mit Sicherheit gelesen und transliteriert werden:

\section{prawijan.haitinaRwas}

Dies sind drei Wörter in urnordischer Sprachform. Die beiden letzten Wörter können ohne weiteres als das Prädikat des Satzes bestimmt werden, als urn. haitinaR was = awn. heitinn vas (var), eine im Altwestnordischen wohlbekannte Fügung. Das erste Wort prawijan kann mit seiner Endung -an jeden obliquen Kasus eines schwachen Maskulinums oder aber den Infinitiv eines Verbs vertreten.

2.2. Die Frage, ob die Inschrift in ihrem erhaltenen Zustand vollständig ist oder ob einige Runen oben weggeschlagen sind, ist mehrmals diskutiert worden (vgl. Stephens 1866-84, 1, 196; Wimmer 1887, 156; Boije 1886, 260; Friesen 1924, 130; Marstrander 1952, 198; Krause 1966, 139).

Sten Boije untersuchte den Stein im Jahre 1883 und stellte fest, daß einige Teile der Oberseite "eine hellere Farbe haben und eine schärfere Kante gegen die Vorderseite bilden, was nach meiner Meinung auf eine Absplitterung deutet. [...] Was am ehesten den Eindruck macht, eine Abspaltung erfahren zu haben, ist der Sektor unmittelbar rechts und danach vielleicht oberhalb der Inschrift." Er neigte deshalb zu der Annahme, daß einige Splitter wälırend des Transportes von Kalleby nach Tanum abgeschlagen sein könnten, glaubte aber nicht, daß nach der Einritzung der Runen und vor dem Bekanntwerden der Inschrift ein umfangreicherer Abschlag erfolgt sein könnte (Boije 1886, 260).

Die Inschrift als solche weist mehrere Züge auf, die auf Vollständigkeit hindeuten. (1) "Während nämlich die Runen in prawijan ziemlich groß und spatiös gestellt sind, werden sie in haitinaR stark gekürzt und zusammengedrängt, um auf dem übriggebliebenen Teil des Steines Platz zu finden. Erst als der Ritzer am Schlusse des Wortes haitinaR erkannte, daß für das letzte Wort genügend Raum 
übrig war, durften die Runen wieder etwas anschwellen" (Friesen 1924, 130; vgl. Moltkes Foto bei Marstrander 1952, 200). (2) Die vierte Rune von hinten, $\psi \mathbf{R}$, hat der Runenmeister so hoch gemacht, daß die Seitenzweige vollständig über die umgebenden Runen hinaufragen; "in this way he saved the distance of nearly a whole letter" (Stephens 1866-84, 1, 201; danach Boije 1886, 261; Friesen 1924, 130). (3) Die letzte Rune $₹ \mathbf{s}$ steht dicht an der Steinkante, ist aber groß und wohlgeformt und in keiner Weise beschädigt. (4) Die Inschrift endet mit einem vollen Wort, und es wäre in Wahrheit ein seltsamer Zufall, wenn eine Bruchlinie genau zwischen zwei Runen und zwei Wörtern verlaufen sollte (vgl. Marstrander 1952, 200). (5) Hinzu kommt ein formales sprachliches Argument. Die Inschrift endet mit einem finiten Verb, und Sätze mit dem Verbum finitum in Endstellung sind ein wohlbekannter Wortstellungstyp im Altgermanischen.

Aus diesen Gründen darf man wohl mit Vollständigkeit der Inschrift rechnen, vorausgesetzt natürlich, daß die drei Wörter sich zu einem syntaktisch korrekten Satz vernünftigen Inhalts verbinden lassen. Das ist denn auch die herrschende Auffassung unter den Runologen seit der Beschreibung des Steins durch Boije 1886. Die Ausnahmen sind Marstrander (Marstrander 1952), der aus sprachlichen Gründen mit dem Verlust eines Wortes nach was rechnete, und Antonsen (Antonsen 1975).

\section{Kritisches Referat der bisherigen Deutungsversuche}

\subsection{Sophus Bugge 1866}

Sophus Bugge meinte (Bugge 1866, $248 \mathrm{ff}$.), das sprachlich nicht ausgedrückte Subjekt des Satzes müsse 'der Stein' sein, und übersetzte: "Er (der Stein) wurde des Thravinge genannt" (indem man damals Rune $6 \sigma$ als $n g$ bestimmt hatte). Gegen diese Deutung können mehrere Einwände erhoben werden.

(1) Der Ausdruck haitinaR was findet sich in jüngerer Sprachform sowohl auf nordischem wie auf westgermanischem Gebiet, vgl. Beispiele wie awn. Knéfrøðr var sá heitinn Akv 1, as. Zacharias uuas hè hêtan $\mathrm{Hel}$ 76, ahd. in thero steti thiu Abilena uuas heizzan Tat 13, 1, aengl. se yldesta was Jared hâten Gen 1063, was his ealdfoder Ecgpeo hāten Beow 373. Das Prädikat dieser Sätze hat die durativ-präteritale Bedeutung 'hieß', d.h. 'trug den Namen'. Demnach wäre für unsere 
Inschrift eine Bedeutung 'Er (der Stein) hieß des Thravinge' anzusetzen. Problematisch daran ist das Präteritum, denn man erwartet ja, daß sich eine Inschrift auf die jeweilige Gegenwart bezieht und in diesem Fall den aktuellen Namen des Steins und nicht seine frühere Benennung angibt. (Vgl. Igijōn hallaR Stenstad KJ 81 'Igjas Stein', das als 'Dies ist der Stein der Igja' zu paraphrasieren ist.)

Um dieser Schwierigkeit zu entkommen nahm Sophus Bugge an, daß haitinaR was nicht 'war genannt', sondern 'wurde genannt' $z u$ übersetzen sei, und daß die Konstruktion dem griechischen Aorist Passiv entspreche (Bugge 1866, 251). Die Bedeutung des Runensatzes wäre demnach: 'Er (der Stein) wurde des Thravinge genannt', d.h. 'er bekam den Namen "des Thravinge (Stein)"'. Von Grienberger machte später (Grienberger 1900, 290) darauf aufmerksam, daß man "im sinne dieser erklärung [...] auf einen besonderen benennungsact schließen [müßte], dessen inhalt und ergebnis in der inschrift mitgeteilt wäre."

Die hier vorgeschlagene Übersetzung ist aber kaum zulässig. In Greins Sprachschatz der angelsächsischen Dichter findet sich nur ein einziger Beleg mit was gehāten (mit präfigiertem Partizip) in punktueller (aoristischer) Aktionsart, und diese Bedeutung ist offenbar kontextbedingt: Crist was on pȳ eahtodan dage Hälend gehāten Menol 4 'Kristus wurde am achten Tag Heiland genannt' (Grein 1912). In den übrigen recht zahlreichen Belegen hat aeng. was häten deutlich durative Bedeutung, und das ist somit auch für das Urnordische als die normale aktionale Interpretation vorauszusetzen. Eine solche Bedeutung paßt aber nicht in den Runentext hinein: Es ist nach wie vor nicht einzusehen, warum der ehemalige Name des Steins bekanntgegeben werden sollte.

(2) Ein weiterer Einwand gegen die Deutung Sophus Bugges besteht darin, daß in den zahlreichen Belegen mit der Fügung aeng. was häten, as. uuas hētan usw. das Prädikativ immer im Nominativ steht; niemals steht es (elliptisch) im Genitiv.

(3) Bugges Deutung setzt eine zweimalige Interpolation des Wortes 'Stein' voraus, erstens als Subjekt, zweitens als Subjektsprädikativ. Ein solcher Satz mit doppelter Ellipse hat keine Parallele in den alten Inschriften, ja kaum noch im ganzen altgermanischen Schrifttum. 


\subsection{Carl Marstrander 1952}

3.2.1. Marstrander übernimmt die von Bugge befürwortete Bedeutung der Fügung haitinaR was und versucht, die oblique Form des Wortes prawijan anders zu erklären. Er betrachtet nämlich die Inschrift als unvollständig und ergänzt sie wie folgt:

prawijan haitinaR was [namne]

'Ich wurde mit dem Namen prawija benannt'.

Syntaktisch ist ein solcher Satz vielleicht möglich. Im Gotischen, Althochdeutschen und Altwestnordischen wird der Dativ des Wortes 'Name' mehrmals so (d.h. instrumental) verwendet, vgl. Marstrander 1952, 202 mit Beispielen wie sah pan haitans was namin Malkus Joh 18,10 'und dieser hieß mit Namen Malkus', then ther anderemo namen hiez Petrum Tat 22,6, Egir, er ९ðru nafni hét Gymir Lok. Prosa, eino nafni hétomk aldregi Grí 48. Die Form prawijan wäre dann als definitiver Genitiv zu erklären (vgl. Nygaard 1905, $\S 123$ mit Belegen wie gáfu Svíar honum Qnundar nafn $\mathrm{Hkr}$ ). Die Verwendung eines definitiven Genitivs bei dem Verb heita ist jedoch eine Seltenheit, die Marstrander nur mit einem Beispiel belegen kann: ok hétom pá hjóna nafni Sigsk 68 'und wir hießen dann mit Namen Eheleute'. Ein Personenname steht jedoch in solchen Fügungen niemals im Genitiv. Im Gotischen steht er immer im Nominativ (drei Belege bei Marstrander 1952, 202), und dafür weist auch das Altwestnordische einen Beleg auf (Lok. Prosa). Die angenommene urnordische Konstruktion ist deshalb nicht unproblematisch.

3.2.2. Marstranders Deutungsvorschlag ist auch mit anderen Schwächen behaftet.

(1) Das unausgedrückte Subjekt des Satzes ist nach Marstrander $e k$ 'ich', d.h. "der Runenmagiker". Seine wichtigste Begründung dafür ist der Name Präwija, dem er die Bedeutung 'der heftige Sehnsucht (awn. prá) verursacht' beilegt. Ein solcher Name könne, meint er, "nur von einem Magiker oder einem übernatürlichen Wesen getragen werden" (Marstrander 1952, 202).

Das Wort soll ein Nomen agentis sein; es kann aber nicht unmittelbar von dem Substantiv awn. prá f 'Sehnsucht' < germ. * prawō abgeleitet sein, denn das würde ein Nomen agentis mit der Bedeutung 'der Sehnsüchtige, jemand, der sich heftig sehnt' ergeben. Um die erwünschte Bedeutung $\mathrm{zu}$ erreichen, postuliert Marstrander ein von 
*brawō 'Sehnsucht' abgeleitetes Verb urn. *bräwijan 'Sehnsucht bewirken', "eine Art Kausativ vom Typ svæfa", wozu ein Nomen agentis urn. * bräwija m 'jemand, der Sehnsucht verursacht' gebildet worden sei. Der Ansatz eines solchen Wortes ist jedoch sehr fraglich: Erstens ist kein Verb awn. *brcefa 'Sehnsucht verursachen' belegt; zweitens ist die Wortbildung (mit Dehnstufe) problematisch, und drittens werden die Nomina agentis auf germ. -jan in ältester Zeit gewöhnlich von Substantiven und nicht von Verben abgeleitet (vgl. Kluge 1926, §1213; Meid 1967, $\S 92,1)$. Marstranders Erklärung dieses Wortes ist deshalb abzulehnen (so auch Krause 1966, 139).

(2) Dadurch ist an der Grundlage seiner These, daß die Subjektperson ein Runenmagiker ist, stark gerüttelt. Hinzu kommt, daß der Runenmeister, wenn er in den alten Inschriften als eigene Person hervortritt, sich klar und deutlich mit dem Pronomen $e k$ und einem Namen oder Epitheton im Nom. Sing. präsentiert (vgl. ek hlewagastiR Gallehus, ek guðija Nordhuglo, ek erilaR Järsberg).

(3) Marstrander beurteilt die Zeitreferenz der Fügung haitinaR was falsch, wenn er die Inschrift mit "Ich wurde mit dem Namen brāwija benannt" übersetzt und daraufhin behauptet, daß dies "in Wirklichkeit" mit \$räwija haite 'ich heiße Prawija' gleichwertig sei (Marstrander 1952, 202). Wie oben gezeigt, hatte die Fügung im Altgermanischen normalerweise durative Aktionsart, bedeutete 'ich/er hieB (so und so)' und mußte sich auf die Vergangenheit beziehen. Diese Inschrift ist somit keine Parallele zu Inschriften mit dem Verb haite im Präsens, wie Müha haite Kragehul, Hariuha haitika Seeland-Br. II.

(4) Marstranders Deutung setzt voraus, daß oben am Stein ein Stück abgeschlagen sei (mit dem Worte namne). Das ist unbeweislich (vgl. oben $\S 2.2$ ), und "nur eine evidente Deutung" könnte eine solche Annahme wahrscheinlich machen, wie es Marstrander selbst ausdrückt (Marstrander 1952, 201).

\subsection{Von Grienberger 1900 und Adolf Noreen 1903, 1923}

Von Grienberger (Grienberger 1900, 294) schlug vor, bei der Deutung dieser Inschrift das Verb awn. heita in der Bedeutung uocari beiseite zu lassen und lieber von dem Verb awn. heita in der Bedeutung 'to promise, vow' auszugehen. Demnach wäre die Inschrift 'Thrauingani devotus sum/est' zu übersetzen, und der Personenname würde im Dativ stehen. Diesen Vorschlag nahm Noreen in die 3. und 4. Ausgabe seiner Grammatik auf (Noreen 1903, 344; Noreen 1923, 389; Noreen 
1970, 389) und übersetzte; "Dem Drefinge/Dem præfe wurde (der Stein) verheißen (oder gewidmet)".

Marstrander hielt das für möglich: "Man kann auch übersetzen: 'er (der Stein) wurde dem Prawija versprochen (oder gewidmet, A. Noreen)', d.h. der Stein mit allem, was er vertritt, den Kult und das damit verbundene Opfer" (Marstrander 1952, 201). Wenn er sich trotzdem für eine andere Interpretation entschied, geschah das, weil der Name prāwija nach seiner Auffassung von einem Runenmagiker zeuge, der als solcher als Subjektperson auftreten müsse.

Religionsgeschichtlich scheint von Grienbergers und Noreens Deutung akzeptabel zu sein. Wir haben gute Gründe für die Annahme, daß der Grabstein als eine Art Altar fungierte, an dem die Kulthandlungen für den Toten verrichtet wurden (vgl. Grønvik 1981, 174 f.) und der deshalb dem Toten gewidmet war. ${ }^{3}$

Sprachlich gibt jedoch diese Deutung zu Bedenken Anlaß; 'einem etwas versprechen' heißt im Altwestnordischen immer heita e-m e-u mit sowohl Personen- wie Sachobjekt im Dativ. Im Passiv ergibt das eine unpersönliche Fügung vom Typ honum var heitit steini. Eine grundlegende Regel der germanischen Syntax besagt nämlich, daß bei der Umbildung eines Aktivsatzes zu einem Passivsatz das Akkusativobjekt des Aktivsatzes zum Nominativsubjekt im Passivsatz wird, während die Genitiv- und Dativobjekte unverändert bleiben (vgl. die Belege aus der altwestnordischen Dichtung bei Grønvik 1985, 125; weiter Behaghel 1923-32, 2, 211; Dal 1962, 130, 168). Diese Regeln für die Aktiv-Passiv-Konversion haben für alle altgermanischen Sprachen Gültigkeit. Dennoch hat niemand bisher an der Syntax des hier besprochenen Deutungsvorschlages Anstoß genommen.

Wahrscheinlich hat man stillschweigend angenommen, daß das Verb urn. *haitan in der Bedeutung 'versprechen' sein Sachobjekt im Akkusativ nahm, wie die entsprechenden präfigierten Verben in Westgermanischen (as. gihētan, ahd. giheizzan, aengl. gehätan). Unter dieser Voraussetzung wäre natürlich eine Passivfügung mit 'er (der Stein)' als Subjekt ohne weiteres möglich.

Hier stehen wir somit der grundlegenden Frage gegenüber, ob wir in den alten Runeninschriften des Nordens eine gemeinsame nordund-westgermanische Sprache suchen dürfen, deren Sprachformen und Fügungsregeln in späterer Zeit sowohl im Westgermanischen wie im Nordischen vertreten sind, oder ob wir davon auszugehen haben, daß

3 Insofern könnte diese Inschrift meine Interpretation und Ergänzung der TuneInschrift unterbauen: (bi-fal)h-k Wōðurīðe staina, vgl. Grønvik 1984, 51-56. 
die Runensprache des 3.-5. Jahrhunderts eine nordische (urnordische) Sprache ist. Diese letztere Ifypothese fordert, daß wir überall dort, wo zwischen dem Westgermanischen und dem Nordischen alte Unterschiede bestehen, in der Runensprache spezifisch nordische Sprachformen und Gebrauchsweisen suchen. Ich habe früher nachzuweisen versucht, daß die Runensprache phonologisch, morphologisch und lexikalisch mehrere nordische Sonderzüge aufweist (Grønvik 1981, 57-69), und finde es deshalb wenig glaubhaft, daß urn. *haitan eine spezifisch westgermanische syntaktische Regel befolgen und das Sachobjekt im Akkusativ $\mathrm{zu}$ sich nehmen sollte, um so mehr als die egentümliche altwestnordische Konstruktion mit zwei Dativobjekten unbedingt alt sein $\mathrm{mu}$.

Auch deswegen halte ich es für notwendig, von Grienbergers und Noreens Deutung abzulehnen.

\subsection{Von Friesen 1924 und Arthur Nordén 1934, 1940}

3.4.1. Einen ganz anderen Weg als die bisher genannten Forscher schlug von Friesen ein. In prawijan sah er eine Schreibung für den Infinitiv urn. *braujan > awn. preyja 'sich sehnen', eine Ableitung von awn. prá $\mathrm{f}<$ urn. * prawō- in der Bedeutung "Sehnsucht, sehnsüchtiges Verlangen, unruhiges Drängen" (Friesen 1924, 131). Weiter behauptete er, daß das Verb urn. *haitan Akkusativ der Person und Infinitiv regieren konnte, und zwar in der Bedeutung 'einem gebieten, etwas zu tun'. Auf diese Weise gelangte er zu dem Passivsatz 'sich zu sehnen wurde er geheißen (wurde ihm geboten)'.

Dies ist nach von Friesen als ein feierlicher Fluch gegen den Grabschänder zu verstehen (Friesen 1924, 134). Die Verwünschung soll bewirken, "daß derjenige, der davon betroffen wird, von Sehnsucht, Mißgedeihen, Unruhe heimgesucht wird [...] und vor mystischen Mächten keine Ruhe findet" (Friesen 1924, 132). Seine endgültige paraphrasierende Übersetzung lautet: "Seine Ruhe und sein Gleichgewicht zu verlieren, in Ängsten zu leben und zu verderben wurde ihm (d.h. dem möglichen Grabschänder) auferlegt" (Friesen 1924, 134).

3.4.2. Nach von Friesen soll das Verb urn. *braujan $>$ awn. breyja

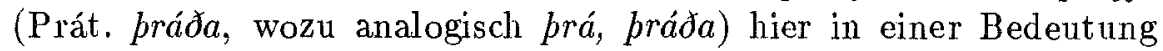
stehen, die man sonst bei den Verben thra und thrana im Mittelschwedischen finden kann, nämlich 'aus Sehnsucht hinschwinden, verschmachten, verderben' (Friesen 1924, 132). Dies fand Nordén unwahrscheinlich: "Diese Bedeutung von isl. preyja muß jedoch mit 
gutem Fug als weniger gebräuchlich bezeichnet werden als die gewöhnliche, die noch heute in dem schwedischen Verb trå hervortritt: ' 1 . sich sehnen, wonach sehnsüchtig verlangen, Heimweh haben [...]' (Rietz). Die Grundbedeutung 'unruhige Sehnsucht' scheint bei trå viel zu stark zu sein, als daß man sich vorstellen kann, daß die Bedeutung 'hinschwinden, verderben' [...] in einer formelhaften Anwendung des Wortes am Anfang des 5. Jahrhunderts die wesentliche gewesen ist" (Nordén 1940, 321 f.).

Nordén schlägt deshalb vor, daß der Fluch gegen den Toten gerichtet ist und ihn hindern soll, das Grab zu verlassen und als Wiedergänger herumzuspuken. Seine Übersetzung lautet: "Sehnsucht (nach dem Grabe zurück) sei [sic, schwed. vare] ihm (dem Beerdigten) auferlegt" (Nordén 1934, 103). Dieser Deutung haben sich später von Friesen (mündlich) und etwas zögernd auch Krause angeschlossen (Krause 1966, 140): "sich zurückzusehnen (nach dem Grabe) war er (der etwaige Wiedergänger) geheißen".

3.4.3. Gegen diese Deutung erheben sich allerlei Zweifel und Einwendungen.

(1) Die Schreibung prawijan für erwartetes * braujan ist sonderbar und hat keine gleichwertige Parallele in harija Skåäng (KJ 85) für sonstiges harja.

(2) Das Verb awn. heita steht mit einem persönlichen Akkusativobjekt in einigen Redewendungen, die von Friesen herangezogen hat: heita e-n út, á brott, heðan, af vist, at rúnum usw. Das Verb hat hier die Bedeutung 'jemanden herausrufen, wegrufen usw.', d.h. 'jemanden bitten oder auffordern herauszukommen, wegzufahren usw.' (vgl. Fritzner 1954, 1, 777). Die Bedeutung des Verbs kommt hier der von von Friesen angegebenen Bedeutung 'einem etwas gebieten' sehr nahe. Voraussetzung dafür ist aber, daß das Verb von einem adverbiellen Glied ut, á brott, heðan oder dgl. begleitet ist, was in dem urnordischen Satz nicht der Fall ist. Auch kann awn. heita in diesen Fügungen mit keinem Infinitiv verbunden werden.

Wenn awn. heita durch einen Infinitiv ergänzt ist, steht das Personenobjekt im Dativ und die Bedeutung ist immer 'versprechen (promise)', vgl. nisl. heita e-m að gera e-t (Blöndal 1920-24, 314), awn. Njáll hét at fara $\mathrm{Nj}$ 49, hétusk peir af haudri Hákon reka Jómsv 12.

Um eine Konstruktion *haitan + Akkusativ der Person + Infinitiv mit der Bedeutung 'einem gebieten, etwas zu tun' für das Urnordische begründen zu können, muß von Friesen deshalb aengl. hătan heranziehen (Friesen 1924, 133, mit zwei Belegen aus Beowulf), das häufig 
so verwendet wird, vgl. heht his engel gān An 365 'er hieß seinen Engel gehen'. Ähnliches gilt für as. hètan (nu hiet hē mē an thesan sìð faran Hel 122) und ahd. heizzan (er hiaz inan irwintan Otfr II 9, 52 'er hieß ihn umkehren').

Ein Durchgang des westgermanischen Materials bringt jedoch an den Tag, daß die entsprechende Passivfügung in alter Zeit nicht belegt ist, und das ist kein Zufall. Diese Konstruktion gehört nämlich zu dem bekannten Typ "Akkusativ mit Infinitiv", der dadurch gekennzeichnet ist, daß der Akkusativ das logische Subjekt des Infinitivs ist. Für solche Fügungen gilt seit alters folgende Regel: "Die Fügungen mit Akkusativ und reinem Infinitiv können im Allgemeinen nicht ins Passiv umgesetzt werden" (Dal 1962, 104). "Der Akkusativ in der Verbindung Akkusativ mit Infinitiv kann nicht zum grammatischen Subjekt gemacht werden" (Dal 1962, 153). Daraus folgt, daß die Konstruktion, die von Friesen, Nordén und Krause für das Urnordische vorausgesetzt haben, syntaktisch nicht möglich ist. Die in der ältesten literarischen Zeit geltende Restriktion in der Anwendung dieses Verbs muß auch für das Urnordische gegolten haben.

\subsection{Elmer H. Antonsen 1975}

Antonsen versucht, das Problem der obliquen Form des Wortes prawijan durch die Annahme zu erklären, daß die Inschrift unvollständig ist und zugleich in zwei Sätze zerfällt:

brawijan. haitinaz was ......

'prawija's (monument). (I,he) was commanded/called ......'

Die elliptische Formel mit dem Namen des Toten im Genitiv (ohne daß ein Wort für 'Stein' oder 'Grab' damit verbunden wäre) findet sich auf den Steinen von Saude (wandarăðas) und Belland (kepan), vgl. Krause 1966, $188 \mathrm{f}$. Hier steht aber der Personenname als einziges Wort auf dem Stein, so daß dem Leser unmittelbar klar wird, daß die Inschrift elliptisch ist.

Ein wort in obliquer Form steht niemals elliptisch, wenn die Inschrift mit anderen Wörtern weitergeht. Ein Personenname im Nominativ kann dagegen syntaktisch isoliert vor einem folgenden Satz stehen, wie etwa IupingaR Reistad und HadulaikaR Kjølvik (sc. 'liegt hier'). Der darauf folgende Satz hat dann immer sein eigenes, sprachlich ausgedrücktes Subjekt, so daß auch hier deutlich angezeigt ist, daß der vorangehende Name einen eigenen Satz bildet. 
Bei dieser Sachlage scheint es mir nicht berechtigt, das erste Wort der Tanum-Inschrift als elliptisch abzutrennen, um mit dem folgenden Wort einen neuen Satz beginnen zu lassen. Übrigens ist die Annahme vom Verlust mehrerer Runen nach dem Worte was sehr unsicher, vgl. oben $\S 2.2$ und $\S 3.2 .2,4$. Über die Übersetzung ' $T$, he was commanded' siehe oben $\S 3.4 .3,2$, über 'I, he was called' oben $\S 3.1,1$ und $\S 3.2 .2,3$.

\section{Eigene Deutung}

4.1. Nach dieser Übersicht über die syntaktischen Probleme, die die Inschrift prawijan haitinaR was bereitet, stellt sich von selbst die Frage: Gibt es etwa im Altwestnordischen Spuren von einer persönlichen Passivkonstruktion mit heita, die vom Typ Knéfrøðr var sá heitinn $\mathrm{Akv} 1(\S 3.1,1)$ verschieden ist?

Im Neuisländischen ist tatsächlich eine solche Fügung im Gebrauch, ist aber auf ein sehr spezielles Verwendungsgebiet beschränkt: sú kona [...] er öðrum manni heitin (Blöndal 1920-24, 314) 'diese Frau ist mit einem anderen Manne verlobt'. Diese Fügung beruht offenbar auf einer Sonderanwendung des Verbs heita in der Bedeutung 'versprechen'. Vielleicht ist sie schon in einer Skaldenstrophe belegt:

pvit eldgrundar Endils

áttgódri mér tródu

betr unnum nú nýtri

nar an heitin veri. Hfr lv 3

'denn ich liebe jetzt die aus edlem Geschlecht stammende, tüchtige Frau (eldgrundar Endils tródu) fast mehr, als wenn sie mir versprochen wäre'

(vgl. die Übersetzung von Finnur Jónsson in SKJ 1912, 1, B, 158).

Dieser Teil der Strophe ist jedoch schlecht überliefert, und die oben angeführte Rekonstruktion Finnur Jónssons ist in mehrerer Hinsicht problematisch. Im altenglischen ist aber eine genau entsprechende Fügung sicher belegt, vgl, sio gehāten (is), geong goldhroden, gladum suna Frōdan Beow. 2024 'sie ist, jung und goldgeschmückt, dem fröhlichen Sohn Frodes versprochen'. Die Übereinstimmung zwischen dem neuisländischen und dem altenglischen Ausdruck deutet auf hohes Alter.

Innerhalb des Nordischen steht diese Ausdrucksweise isoliert da, denn im Aktiv fordert das Verb awn. heita zwei Objekte im Dativ, auch wenn beide Objekte Personen sind, vgl. Hefir minn faðir meyjo 
sinni grimmom heitit Granmars syni $\mathrm{HH}$ 1, 18 'mein Vater hat seine Tochter dem grimmigen Sohn Granmars versprochen'. Im Passiv wäre deshalb eine unpersönliche Konstruktion zu erwarten, und dafür kann man in der Prosa auch Belege finden, wie kom svá, at Bárði var heitit meyjunni Eg 7. Wenn nun eine persönliche Passivfügung mit spezialisierter Bedeutung daneben steht (nisl. hón var öðrum manni heitin), muß diese eine ältere Konstruktion vertreten (heita + Akkusativ der Person + Dativ der Person), die im Aktiv früh aufgegeben wurde und sich nur in passivischen Partizipialkonstruktionen erhalten hat.

In der Inschrift von Tanum haben wir es offenbar mit einer derartigen Passivfügung zu tun. Diese muß ein personanzeigendes Subjekt haben und kann nur bedeuten: 'Dem Prawija war (ich/er) versprochen'.

4.2. Was bedeutet denn prawija, -an m? Das Wort läßt sich unschwer als eine Ableitung zu awn. prá f < urn. *brawō- 'Sehnsucht' erklären. Es ist mit einem Suffix - jan gebildet, das zur Bildung von Nomina agentis diente und gerade im ältesten Nordischen produktiv war (vgl. Kluge 1926, § 12-13; Meid 1967, § 92, 1). Die Suffixvariante -ijan trat hier ein, weil dadurch die phonemische Struktur der Wurzel unverändert bleiben konnte. Die Bildung ist also vom Urnordischen aus gesehen durchsichtig und klar; das Wort kann nur bedeuten 'jemand, der sich (heftig) sehnt, der Sehnsüchtige'.

$\mathrm{Zu}$ bemerken ist, daß das Wort awn. prá $\mathrm{f}$ häufig von der Sehnsucht des Mannes nach einer Frau verwendet wird, vgl. pró muna oss of avi eldask Korm lv 2 'meine Sehnsucht (nach dir) wird niemals veraltern' (ähnlich Mhkv 13, 27, Sigsk 7, Fj 50).

Wenn die Inschrift statt haitinaR die Form *haitinu gehabt hätte: prawijan *haitinu was, würde die Subjektperson eine Frau sein: '(sie) war dem Sehnsüchtigen versprochen'. Ein solcher Satz würde den oben $\S 4.1$ zitierten isländischen und altenglischen Belegen genau entsprechen und einen netten Sinn haben: Zeugnis einer glücklichen Verlobung. Die Form haitina $R$ setzt aber voraus, daß die Subjektperson männlich ist, wie auch prawija. Auf welche Situation zeigt dies hin? Wird im nordischen Kulturkreis ein Mann jemals einem anderen Mann oder einem Wesen männlichen Geschlechts versprochen?

4.3. In Saxos Erzählungen, in Fornaldarsagas und in Märchen hat Ot to Höfler (Höfler 1952) Spuren einer alten Glaubensvorstellung gefunden, die darin besteht, daß ein Ehepaar vor der Empfängnis oder vor der Geburt ihr Kind einem Gott versprechen (geben, weihen) konnte. Dieser Gott würde seinen Verehrer in Leben schützen und ihn nach 
dem Tode zu sich nehmen. Besonders von Odin wird dies berichtet. Er half seinen Schützlingen im Leben, ließ sie aber gnadenlos fallen, wenn ihre Zeit zu Ende war, um sie in sein Totenreich aufzunehmen. Dies nennt Höfler "die germanische Individualweihe" und hält es für eine wichtige Institution der altgermanischen Religion.

Ein so kritischer Forscher wie Hans Kuhn rechnet auch mit einer solchen religiösen Praxis: "Neben der Weihung von Feinden an den Kriegs- und Totengott hat es auch die ganz andere gegeben, mit der man einem Gotte Freunde zu eigen gab, um ihnen seinen besonderen Schutz zu sichern [...]. Diese Weihung lebt in der Überlieferung hauptsächlich in der Schicht der Sage und ist deshalb nicht ernst genommen, doch hat jetzt Otto Höfler ihre Bedeutung stark herausgearbeitet" (Kuhn 1954, 427; Kuhn 1971, 372). Anne Holtsmark sieht ebenfalls hier "mehr oder minder vage Erinnerungen an heidnischen Kult" (Holtsmark 1953, 145), und sogar Höflers Antagonist in dem Streit um die Rök-Inschrift, Elias Wessén, gibt zu, daß dies "religionsgeschichtlich sicherlich von bedeutendem Interesse" ist (Wessén 1953, 173).

4.4. In einem Aufsatz von 1983 habe ich zu zeigen versucht, daß diese religiöse Praxis der dunklen Schlußpartie der Rök-Inschrift zugrundeliegt. Einleitend werden (in der Form von minni 'Erinnerungen', d.h. 'Gedächtnissprüche') zwei Fragen gestellt, die ich hier in normalisierter altwestnordischer Sprachform wiedergebe, zuerst:

hverr Ingoldinga vœri goldinn at kvánar húsli?

'wer von den Nachkommen Ingvalds bei der Opferhandlung der Frau (einem Gotte) gegeben ${ }^{4}$ wäre?'

und danach:

hveim sé borinn niðr drengi?

'welchem tapferen Burschen ein Verwandter (awn. niðr) geboren sei?'

Die Antwort auf diese letzte Frage verteilt sich auf zwei Aussagen (minni). Zuerst wird die Identität des tapferen Burschen durch seine Heldentaten angedeutet:

4 Den Ansatz dieser Bedeutung des Verbs awn. gjalda, Part. goldinn werde ich in einer späteren Arbeit näher begründen. 


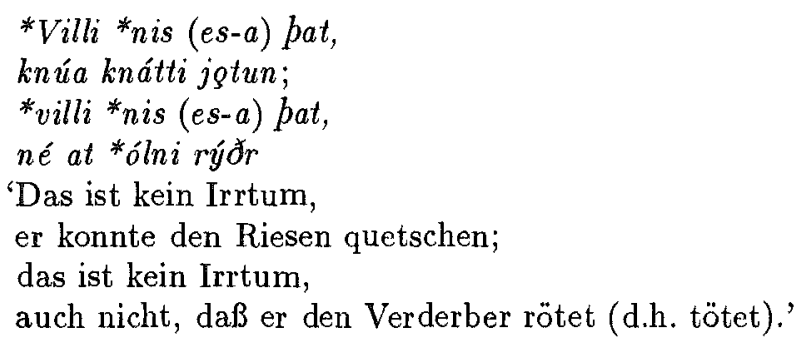

Danach wird sein in komplizierten Runen verborgener Name wörtlich ausgedrückt und von hymnischen Attributen begleitet, die zugleich den Höhepunkt und den Schluß der Inschrift bilden:

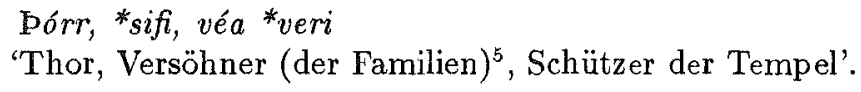

Die Antwort auf die Frage, wem ein Verwandter geboren sei, ist also; dem Gotte Thor. Und die Antwort auf die erste Frage, wer bei der Opferhandlung der Frau (dem Gotte) gegeben worden sei, kann nur sein: der Sohn Væmod, zu dessen Verehrung und Andenken die ganze Runeninschrift hergestellt wurde. Weil er bei der Opferhandlung "der Ehefrau" (d.h. der Ehefrau Varins, des Runenritzers) dem Gotte Thor gegeben (versprochen) wurde, wird er als dessen Verwandter, als pórs niðr geboren. Ähnlich ist wohl auch die Tatsache zu erklären, daß Hákon jarl als Yggs nidr (Vell 20) und ragna konr (Vell 32) und daß Könige und Häuptlinge in Ynglingatal und Háleygjatal als Freys oder Týs óttungr bezeichnet werden: Vor ihrer Geburt waren sie schon durch ein religiöses Rituale einem Gotte zugeeignet.

Bei dieser Deutung erweist sich die Rök-Inschrift als ein wichtiges religionsgeschichtliches Dokument, das die in Frage stehende religiöse Praxis für die frühe Wikingerzeit sicherstellt. ${ }^{6}$

${ }^{5}$ Sifi läßt sich besser so erklären denn als 'Gatte der Sif' (Grønvik 1983, 128). Das Wort ist wohl, wie auch *veri (1.c. 127), ein Nomen agentis, kurzsilbig und deshalb analogisch gebildet. Es gehört zu einem später verlorenen Verb, das als got. gasibjon, aengl. (ge) sibbian 'versöhnen' gut belegt ist, vgl. aber auch awn. sifjaðr 'durch Schwägerschaft verbunden, verwandt'. Ursprünglich bedeutete es offenbar 'zwei Familien durch Heirat verbinden und dadurch versöhnen'. Eine solche Funktion kann ohne Schwierigkeit dem Gotte Thor zugetraut werden, vgl. seine Weihefunktion in der Prymskviða.

${ }^{6}$ Die durchgehende und alles verbindende Formel sakunıkmini $=$ sggum ' $k$ minni 'ich sage einen Gedächnisspruch (eine kurze Gedenkrede) auf' und ihre Beziehung zu dem jüngeren Ausdruck mala fyrir minni, drekka minni $(\epsilon-s)$ werde ich in einem späteren Aufsats näher erörtern. 
4.5. In einer vor kurzem erschienenen Arbeit (Grønvik 1987) habe ich den Anfang der Inschrift von Stentoften (KJ 96) behandelt und darin die epische Einleitung zum folgenden Grabschutzformular gesucht. Nach erneuten Überlegugen zur Lesung und Deutung der z. T. beschädigten Runen lege ich hier eine etwas modifizierte Deutung vor, die mit der traditionellen Lesung der Runen besser übereinstimmt ${ }^{7}$ :

\section{niu hA-borumR \\ niu ha-gestumR}

hA pu-wolAfR gAf

$A(h) h A$ riwol AfR $\mathbf{m A g}(i)$ usnu hle

'Neun $h \bar{a}$-Söhnen, neun $h \bar{a}$-Gästen

Hálfr opferte (sc. Beigaben);

gab Herjolfr dem Sohne einen Grabhügel aus Feuer.'

Einige Sprachformen bedürfen eines kurzen Kommentars. Da das Verb awn. gefa in sakraler Verwendung in der Bedeutung 'opfern, weihen' belegt ist (Beck 1967, 124; Düwel 1984, 148), kann es auch hier in dieser Bedeutung (aber ohne Objekt) stehen. In dem Worte /ah/ sehe ich nicht mehr (wie Grønvik 1987, 125) eine alte Konjunktion, sondern das Präteritum des starken Verbs urn. *jehan, jah (= as., ahd. jehan) mit lautgesetzlichem Schwund des anlautenden $j$-. Eine neugebildete Präsensform iAr $=j \bar{a} R$ liegt auf der Spange von Fonnås vor und zwar in der Bedeutung 'geben, übertragen' (Grønvik 1987, 41); dort wie hier regiert das Verb Dativ der Person + Dativ der Sache. In mAgi steckt das Wort /mægi/ = awn. megi 'dem Sohne'; nach der $u$-Synkope um 550 war das die lautgesetzliche Dativform (< urn. ${ }^{*}$ magiu). In hle sehe ich eine Schreibung für $/$ hlæ/ $=$ awn. hla < urn. hlaiwa (Bø, KJ 78), got. hlaiw usw. 'Grab, Grabhügel'. In literarischer Zeit ist dieses Wort vermutlich in den Odinsnamen Hlakfg $\partial r$ und Hlakfreyr belegt (Noreen 1923, $\S 97,3$; Vries 1962, 236), sonst nicht. (In dieser Inschrift bezeichnet die Rune $M$ e somit nicht nur die Phoneme /e,e:/, sondern auch /æ,æ:/: ein kurzes /æ/ in gestumR $=/$ gæstumR/ und ein langes $/ æ: /$ in hle $=/$ hlæ: $/$. Vor dem erhaltenen Umlautsfaktor wird dagegen $/ æ /$ als $*$ A notiert, vgl. $\mathbf{m A g i}=/$ mægi $/$ und hAri $=/$ hæri $/$ wie auch hAbu $=/$ hopl $/$.)

\footnotetext{
7 In einem Brief vom 15. Dezember 1987 teilte mir Marie Stoklund mit, daß sie nach Autopsie im Sommer 1987 die herkömmliche Lesung von Rune IV 11 als $\ \mathrm{~m}$ bevorzuge und meine Lesung der Rune IV 13 als $\nvdash$ k nicht akzeptieren könne; die erhaltenen Spuren liessen eher auf eine Rune $X \mathrm{~g}$ schließen.
} 
Das nur hier belegte Wort usnu $=/$ ysnu $/$ kann nur Dat. Sg. Ntr. eines Adjektivs awn. ${ }^{*} y \sin n<$ urn. ${ }^{*} u \operatorname{sina} R$ sein, in dem man ein mit dem Suffix -ina-gebildetes Stoffadjektiv zum Substantiv awn. ysja f. 'Feuer' < urn. *usjō erkennt (vgl. Vries 1962, 636). Das Syntagma

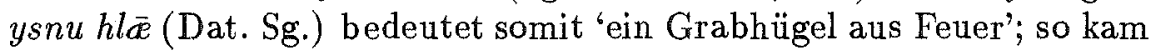
ihnen der hoch emporflammende Scheiterhaufen mit den Leichen der Verstorbenen und den vielen Beigaben vor. ${ }^{8}$

Die Inschrift berichtet, daß Hálfr und Herjolfr neun Männer ehrenvoll beerdigt haben. Von diesen neun wird einer als der Sohn des Herjolfr bezeichnet; vermutlich war er der Anführer der neun Männer. Auch Hálfr wird ein naher Verwandter des verstorbenen Häuptlings gewesen sein, etwa der Großvater, der Onkel oder der Bruder.

Einen Anhaltspunkt für eine nähere Beschreibung der neun Männer haben wir in ihrer Bezeichnung als ' $h \bar{a}$-Söhne' und ' $h \bar{a}$-Gäste'. Nehmen wir an, daß das Adjektiv awn. há-r 'hoch' hier in der Bedeutung 'sozial hochstehend, vornehm' steht, geraten wir in große Schwierigkeiten. Die neun Männer können ja nicht zugleich die Söhne und die Gäste des Herjolfr sein - seine Söhne würde Herjolfr nicht zugleich als Gäste bezeichnen; übrigens scheint er nur einen Sohn unter den Gefallenen zu haben. Bei der Annahme, daß die übrigen acht Männer die Söhne des IIálfr sind, versteht man nicht, warum nicht auch seine Vaterschaft angedeutet wird; und wie erklärt man dann die 'Gäste'? Daß die Benennungen 'Söhne' und 'Gäste' sich auf respektive Herjolfr und

${ }^{8} \mathrm{Da}$ es sich hier um die Weihe eines Grabes handelt, bestätigen die folgenden Worte hideRrunono $=h \bar{i} \partial^{e} R$ rünono 'das Lager der vertrauten Freunde', die ich jetzt (anders als Grønvik 1987, 128) als den Anfang der folgenden Grabschutzformel betrachte. Das Wort rūnonō ist der regelmäßige Gen. Plur. eines schwachen Maskulinums, das als awn. rúni m 'vertrauter Freund' weiterlebt; seiner Herkunft nach ( $<$ germ. * ga-rūnan-) bezeichnet es eigentlich Männer, die Geheimnisse miteinander teilen, was ja für diese neun Männer vortrefflich paßt, vgl. unten über ihre OdinWeihe.

Das Wort $h \bar{i} \partial^{e} R$ ist ein ursprünglicher es-Stamm, der später sein $-R$ analogisch

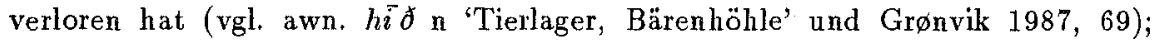
es scheint hier in einer älteren Bedeutung 'Lager, Ruhestätte (für Menschen)' vorzuliegen, von der es auch im Altwestnordischen Spuren gibt (Gronvik 1987, 128). Neben dem alten es-Stamm run. $h \bar{i}{ }^{e} R(>$ awn. hí $\partial$ ) stand eine j-Ableitung urn. *hiððija n, seit etwa $500^{*} h \bar{i} \partial i$ (> awn. hiði, altschw. hipe). Es ist nun möglich, daß finn. hiisi, obl. hïde-, dessen äl teste Bedeutung 'burial place' ist (Koski 1977), als Lehnwort auf dieses nordische Wort zurückgeht, vgl. den Beitrag von Mauno Koski in diesem Band der SIDA. Das wäre eine starke Stütze für die oben angesetzte Bedeutung des runischen Wortes. Die Lehnwort-Theorie ist jedoch nicht ganz sicher, da für das finnische Wort auch eine andere Erklärung in Betracht kommt, siehe Koski 1977. 
Hálfr beziehen sollte, ist syntaktisch unmöglich.

Um von diesen Schwierigkeiten loszukommen, müssen wir die Bezeichnungen ' $h \bar{a}$-Söhne' und ' $h \bar{a}$-Gäste' mit dem Odinsnamen *Hávi, obl. Háva 'der Hohe' (Hávm 109, 111, 164) verbinden; urn. *hauha$>h \bar{a}$ - ist die alte Kompositionsform sowohl der starken wie der schwachen Form des Adjektivs.

Die neun Männer werden also zuerst ' $h \bar{a}$-Söhne' = 'Odins-Söhne' genannt. Das besagt wohl, daß sie schon vor ihrer Geburt dem Odin zugeeignet waren und deshalb als seine Söhne angesehen wurden, ganz wie Væmod in der Rök-Inschrift als der Verwandte Thors galt. Demzufolge haben wir es hier mit Odin-Kriegern zu tun, die vermutlich gleichzeitig im Kampf gefallen waren und hier eine gemeinsame Grabund Kultstätte bekamen.

Wenn sie danach ' $h \bar{a}$-Gäste' = 'Odins-Gäste' benannt werden, kann das nur bedeuten, daß sie jetzt Odin in Walhall besuchen werden. Die Bezeichnungen $h \bar{a}$-borum $R$ und hă-gestum $R$ sind es also, die - in dieser Reihenfolge - der Inschrift ihre religiöse Perspektive verleihen. Hier spürt man die feste Überzeugung der Odin-Verehrer, daß die ihm geweihten Krieger nach dem Tode in sein Totenreich aufgenommen werden. Diese zentrale Glaubensvorstellung der altnordischen Religion kommt hier zum ersten Mal in einem schriftlichen Denkmal zum Ausdruck.

4.6. Auf die beiden Inschriften von Rök und Stentoften gestützt, können wir jetzt auch den Sinn der Tanum-Inschrift begreifen: prawijan haitinaR was 'dem Prawija war (er) versprochen'. In Prawija muß der Name eines Gottes stecken, und das unausgedrükte Subjekt des Satzes kann sich wohl nur auf den Toten beziehen, dem der Stein errichtet wurde. ${ }^{9}$ Hier ist also ein heidnischer Grab- oder Gedenkstein, der von dem innigen Verhältnis des Toten zu seinem Gotte zeugt.

4.7. Prawija ist also das heiti (der Deckname) eines Gottes, der durch

${ }^{9}$ Setzt man versuchsweise den Runenritzer ( $e k$ 'ich') als Subjekt an, stört das Tempus Präteritum. Denn von einem lebendigen Menschen erwartet man ja, daß er von sich selbst sagt: 'Ich bin einem Gotte versprochen (geweiht)'. Mit Präteritum würde der Satz besagen, daß er sich jetzt von seinem Gottesverhältnis losgelöst hätte. Dagegen kann eingewendet werden, daß Passivfügungen mit vas (var) im Altwestnordischen oft mit 'wurde' übersetzt werden können, und ein Satz wie 'ich wurde einem Gott versprochen' könnte auch von einem lebendigen Gottesverehrer ausgesprochen sein. Bei der Annahme eines 'ich' als Subjekt würde also der Satz gerade im Hinblick auf das Gottesverhältnis doppelsinnig sein, und das paßt doch sehr schlecht auf einem Grab- oder Gedenkstein. 
diesen Namen als 'der Sehnsüchtige, der sich (heftig) Sehnende' bezeichnet wird. Gibt es einen frühnordischen Gott, der wegen seiner (heftigen) Sehnsucht so bekannt ist, daß ein solcher Name ihn charakterisieren und identifizieren würde?

Eine in literarischer Zeit wohlbekannte Mythe handelt von dem Gotte Freyr, dem Sohn des Njgrðr, der eines Tages eine überaus schöne Frau in den Jotunheimar erblickte; das war Gerðr, die Tochter des Riesen Gymir. Par af fekk hann hugsóttir miklar heißt es in der Prosaeinleitung der Skírnismál, und in dem Gedicht wird berichtet, daß er ofreidr 'sehr zornig' war, weil er sie nicht haben durfte. Tagelang saß er allein in seinem Haus með mikinn móðtrega 'mit großem Kummer im (aufgeregten) Sinn' (Skí 3).

Sein Diener Skírnir fährt dann nach den Jotunheimar, um für ihn zu werben, und durch Drohungen erzwingt er ihr Gelïbde, innerhalb von neun Tagen dem Gott im Hain Barri zu begegnen: "dort wird Gerðr dem Sohn des Njorðr Freude gönnen." Als Freyr dies erfährt, antwortet er mit den berühmten Worten:

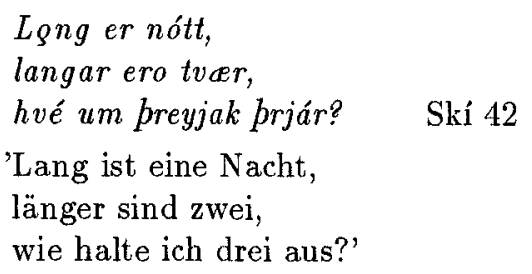

Von Anfang bis Ende ist also Freyr in diesem Gedicht als der von sehnsüchtigem Verlangen nach der Riesentochter erfüllte Gott dargestellt. Es ist zwar möglich, daß gewisse alte mythische Elemente in diesem Gedicht literarischen Zwecken angepaßt sind; wahrscheinlich ist jedoch, daß das Motiv der leidenschaftlichen Sehnsucht altererbter Mythenstoff ist und zum traditionellen Bild des Fruchtbarkeitsgottes gehörte - es paßt sehr gut zur Vorstellung von einem männlichen Fruchtbarkeitsgott.

"Hinter der Mythe von Freyr und Gerðr liegt wahrscheinlich ein rituelles Frühlingsfest, in dem die 'heilige Hochzeit' zwischen dem Gott und der Erdgöttin mit Prozessionen gefeiert wurde, wie sie Tacitus in seiner Schilderung der Erdgöttin Nerthus und ihres Kults beschreibt" (Halvorsen 1980-82, 4, 618). Die Vorstellung von dem Fruchtbarkeitsgott und seiner leidenschaftlichen Sehnsucht nach der Erdgöttin bzw. der Riesentochter wird deshalb eine zentrale, alte und weithin verbreitete religiöse Vorstellung gewesen sein. Darauf deutet auch die 
bildhafte Darstellung des Freyr cum ingenti priapo, die sich laut Adam von Bremen (um 1070) im Tempel von Uppsala befand. ${ }^{10}$

Die Personenbezeichnung prawija 'der Sehnsüchtige' kann deshalb kaum auf einen anderen Gott als Freyr bezogen werden, wenn sie wie hier in einem Kontext erscheint, der gerade eine Bezeichnung für einen Gott erwarten läßt.

4.8. Der Inhalt der Tanum-Inschrift ist somit, daß der Tote, dem der Stein errichtet wurde, dem Fruchtbarkeitsgotte Freyr versprochen war. Daraus folgt von selbst, daß er ihm auch im Leben gedient hatte. Was wir aus den Inschriften von Rök und Stentoften herauslesen können, weist auf die weitere Vorstellung hin, daß er nach dem Tode zu seinem Gott gekommen war. Dadurch wird verständlich, wieso man die Inschrift auf seinem Grabstein (oder Gedenkstein) so knapp formulieren konnte. In ihrer gedrängten Form zeugt sie von heidnischer Glaubensgewißheit: Man wußte, daß der Tote dem Gott versprochen war; es erübrigte sich hinzuzufügen, daß er nun auch bei seinem Gotte angelangt war.

\section{Schlußbemerkungen}

Oben haben wir drei nordische Runeninschriften besprochen, die von der alten heidnischen Sitte zeugen, ein noch ungeborenes $\mathrm{Kind}$ einem Gotte zu geben bzw. zu versprechen, d.h. durch ein religiöses Ritual es dem Gotte zuzueignen. Diese religiöse Institution, von der Otto Höfler bei Saxo, in Fornaldarsagas und in Märchen noch Spuren fand und die er "die germanische Individualweihe" nannte, ist somit für die ältere nordische Zeit sicher bezeugt.

Festzustellen ist nun, daß man allen drei Hauptgöttern des Nordens, dem Thor, dem Odin und dem Freyr Schützlinge weihte. Daß es sich bei Saxo und in den Fornaldarsagas nur um Weihungen an Odin handelt, wird darauf beruhen, daß Odin im Spätheidentum gerade bei Kriegern und Skalden hohes Ansehen und große Beliebtheit gewonnen hatte und deshalb in literarischen Quellen häufig erwähnt wird.

Die betreffenden Runensteine sind unterschiedlichen Alters. Der Rök-Stein ist auf die frühe Wikingerzeit (800-820), der Stein von Stentoften auf das 6. Jahrhundert (550-580/600) und der Stein von Tanum mit einer rein urnordischen Inschrift auf den Zeitraum 200-500 zu

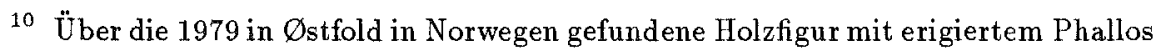
aus der Zeit um 300 siehe Johansen 1985. 
datieren. Die Formen der Runen $\mathbf{j}, \mathbf{R}$ und $\mathbf{w}$ sowie die Schriftrichtung von rechts nach links passen in den letzten Teil dieser Epoche nicht gut hinein. Krause datiert die Inschrift auf die Zeit um 400, und das mag das Richtige treffen. Die erwähnte religiöse Praxis erstreckt sich also von der späten Römerzeit bis in die frühe Wikingerzeit hinein.

Diesen Runensteinen müssen wir somit eine religiöse Funktion zuerkennen. Diese Möglichkeit ist wohl auch bei der Interpretation anderer alter Runeninschriften in Betracht zu ziehen.

\section{Literaturverzeichnis}

Antonsen, E. H. 1975. A concise grammar of the older runic inscriptions. Tübingen.

Bæksted, A. 1951. Begravede Runestene. Aarbøger for nordisk Oldkyndighed og Historie. København.

Beck, I. 1967. Studien zur Erscheinungsform des heidnischen Opfers nach altnordischen Quellen. München.

Behaghel, O. 1923-32. Deutsche Syntax 1-4. Heidelberg.

Blöndal, S. 1920-24. Islandsk-Dansk Ordbog. Reykjavík.

Boije, S. 1886. Bidrag till Kännedom om Göteborgs och Bohusläns Fornminnen och Historia 3. Stockholm.

Bugge, S. 1866. Bidrag til Tydning af de ældste Runeindskrifter. Tidskrift for Philologi og P\&dagogik 7. Kjøbenhavn.

Dal, I. 1962. Kurze deutsche Syntax auf historischer Grundlage. Tübingen.

Düwel, K. 1984. Das Opferfest von Lade. (Wiener Arbeiten zur germanischen Altertumskunde und Philologie 27.) Wien.

Friesen, O. v. 1924. Rö-stenen i Bohuslän och runorna i Norden under folkvandringstiden. (Uppsala universitets årsskrift 1924.) Uppsala.

Fritzner, J. 1954. Ordbog over det gamle norske Sprog 1-3. Oslo.

Grein, C. W. M. 1912. Sprachschatz der angelsächsischen Dichter. Unter Mitw. von F. Holthausen neu hrsg. von J. J. Köhler. Heidelberg.

Grienberger, Th. v. 1900. Neue Beiträge zur Runenlehre. Zeitschrift für deutsche Philologie 32. Halle.

Grønvik, O, 1981. Runene på Tunesteinen. Oslo.

- 1983. Runeinnskriften på Rök-steinen. Maal og Minne. Oslo.

- 1984. Svar fra doktoranden. Maal og Minne. Oslo.

- 1985. Runene på Eggjasteinen. Oslo.

- 1987. Fra Agedal til Setre. Oslo.

Halvorsen, E. F. 1980-82. Freyr. Kulturhistorisk leksikon for nordisk middelalder 4. [København].

Holtsmark, A. 1953. Rez. Höfler 1952. Maal og Minne. Oslo. 
Höfler, O. 1952. Der Runenstein von Rök und die germanische Individualweihe. (Germanisches Sakralkönigtum 1.) Tübingen.

Johansen, Ø. 1985. Jernalderguden i Skjeberg. Det Norske VidenskapsAkademi. Årbok. Oslo.

Kluge, F. 1926. Nominale Stammbildungslehre der altgermanischen Dialekte. 3. Aufl. bearb. von L. Sütterlin und E. Ochs. Halle (Saale).

Koski, M. 1977. The change of semantic structure in some Balto-Finnic place names. Onoma 21, 1-2. Louvain.

Krause, W. 1966. Die Runeninschriften im älteren Futhark. Göttingen.

Kuhn, H. 1954. Gaut. Festschrift für Jost Trier. Meisenheim/Glan.

- 1971. Kleine Schriften 2. Berlin.

Marstrander, C. 1952. De nordiske runeinnskrifter i eldre alfabet. Viking 16. Oslo. [Offprint.]

Meid, W. 1967. Wortbildungslehre. (Germanische Sprachwissenschaft 3.) Berlin.

Nordén, A. 1934. Från Kivik till Eggjum 2. Fornvännen 29. Stockholm.

- 1940. Tysk runforskning under de sista åren. Fornvännen 35 . Stockholm.

Noreen, A. 1903. Altisländische und altnorwegische Grammatik unter Berücksichtigung des Urnordischen. 3. vollst. umgearb. Aufl. Halle.

- 1923. Altisländische und altnorwegische Grammatik unter Berücksichtigung des Urnordischen. 4. vollst. umgearb. Aufl. Halle.

- 1970. Altisländische und altnorwegische Grammatik unter Berücksichtigung des Urnordischen. 5. unveränd. Aufl. Tübingen.

Nygaard, M. 1905. Norrøn Syntax. Kristiania.

SKJ Den norsk-islandske Skjaldedigtning 1, A-B. 1912. [Ed. by] F. Jónsson. København.

Stephens, G. 1866-84. The Old-Northern runic monuments of Scandinavia and England 1-3. London.

Vries, J. de. 1962. Altnordisches etymologisches Wörterbuch. Leiden.

Wessén, E. 1953. Nytt om Rök-stenen. Fornvännen 48. Stockholm.

Wimmer, L. 1887. Die Runenschrift. Berlin. 